Enferm Bras 2019;18(3):359-64

https://doi.org/10.33233/eb.v18i3.2228

\title{
ARTIGO ORIGINAL \\ Internações hospitalares por dependência química no Brasil: um estudo epidemiológico
}

Raiane Jordan da Silva Araújo*, Janaína Paula Calheiros Pereira Sobral, M.Sc. ${ }^{* *}$, Roberta Virtuoso de Souza**, Yasmin Virtuoso de Souza***

*Enfermeira, Especialista em Saúde Mental, Especialista em Gestão em Saúde Pública, Hospital Universitário Professor Alberto Antunes, Maceió/AL, ${ }^{* *}$ Enfermeira, Mestra em Enfermagem, Universidade Federal de Alagoas/UFAL, Maceió/AL, ${ }^{* * * E n f e r m e i r a, ~ E s p e c i a l i s t a ~ e m ~ O n c o l o g i a, ~}$ Faculdade SEUNE/AL, ${ }^{* * * * A c a d e ̂ m i c a ~ d e ~ E n f e r m a g e m, ~ U n i v e r s i d a d e ~ E s t a d u a l ~ d e ~ C i e ̂ n c i a s ~ d a ~}$ Saúde de Alagoas/UNCISAL, Maceió/AL

Recebido em 26 de março de 2018; aceito em 10 de janeiro de 2019.

Correspondência: Raiane Jordan da Silva Araújo, Hospital Universitário Professor Alberto Antunes, setor de Radiologia, Av. Lourival Melo Mota, S/N Tabuleiro do Martins 57072-970 Maceió AL, E-mail: raianejsa@hotmail.com; Janaína Paula Calheiros Pereira Sobral: nainacalheiros2@gmail.com; Roberta Virtuoso de Souza: robertav.souza@hotmail.com; Yasmin Virtuoso de Souza: yasminvirtuoso@hotmail.com

\section{Resumo}

Esta pesquisa teve como objetivo analisar os dados epidemiológicos acerca das internações hospitalares por transtornos mentais e comportamentais decorrentes do abuso do álcool e de outras substâncias psicoativas no Brasil, de 2007 a 2016. Configura-se uma pesquisa descritiva de corte transversal com abordagem quantitativa baseada em dados documentais oriundos do Sistema de Informações Hospitalares/DATASUS. Os percentuais analisados e discutidos foram: ano, sexo, idade e região. Houve redução de mais de $30 \%$ na quantidade de internações no período de 2011 a 2016, com predominância no sexo masculino, faixa etária de 30 a 49 anos, em São Paulo e Região Sudeste. O presente estudo constatou que houve impacto positivo na saúde pública no sentido da desinstitucionalização nos tratamentos em saúde mental e que o público feminino e a região sudeste precisam ser alvos de mais ações em relação ao uso do álcool e de outras substâncias psicoativas e a redução de danos.

Palavras-chave: transtornos relacionados ao uso de substâncias, hospitalização, saúde mental.

\begin{abstract}
Hospitalizations by chemical dependency in Brazil: an epidemiological study

This study aimed to analyze the epidemiological data about hospital admissions due to mental and behavioral disorders resulting from alcohol and other psychoactive substances abuse in Brazil from 2007 to 2016. A descriptive cross-sectional study with a quantitative approach based on documentary data from the Hospital Information System / DATASUS. The percentages analyzed and discussed were: year, sex, age and region. There was a reduction of more than $30 \%$ in the number of hospitalizations in the period from 2011 to 2016, with a predominance of males, aged between 30 and 49 years, in São Paulo and Southeast Region. The present study found that there was a positive impact on public health in the sense of deinstitutionalization in mental health treatments and that the female audience and the southeast region need to be targets of more actions regarding the use of alcohol and other psychoactive substances and the reduction of damage.
\end{abstract}

Key-words: disorders related to substance use, hospitalization, mental health.

\section{Resumen}

Internaciones hospitalarias por dependencia química en Brasil: un estudio epidemiológico Esta investigación tuvo como objetivo analizar los datos epidemiológicos acerca de las internaciones hospitalarias por trastornos mentales y comportamentales derivados del abuso del alcohol y de otras sustancias psicoactivas en Brasil, de 2007 a 2016. Se configura una investigación descriptiva de corte transversal con abordaje cuantitativo basado en datos documentales provenientes del Sistema de Informaciones Hospitalarias / DATASUS. Los 
porcentuales analizados y discutidos fueron: año, sexo, edad y región. En el período de 2011 a 2016, con predominio en el sexo masculino, grupo de edad de 30 a 49 años, en São Paulo y Región Sudeste hubo reducción de más del $30 \%$ en la cantidad de internaciones. El presente estudio constató que hubo un impacto positivo en la salud pública en el sentido de la desinstitucionalización en los tratamientos en salud mental y que el público femenino y la región sudeste necesitan de ser objeto de más acciones en relación al uso del alcohol y de otras sustancias psicoactivas y la reducción de daños.

Palabras-clave: trastornos relacionados con el uso de sustancias, hospitalización, salud mental.

Introdução

O uso de drogas é um fenômeno utilizado com um recurso que acompanha o ser humano ao logo de toda a história não se reduzindo a uma relação linear de causa e efeito, mas inserese numa perspectiva complexa, por envolver fatores pessoais e também políticos e sociais [1,2].

O processo de adoecimento psíquico decorrente do uso de drogas, independentemente de ser devido ao uso do álcool ou de outras substâncias psicoativas, podem levar ao usuário a necessidade de um tratamento através da internação hospitalar. Mesmo desconsiderando, em alguns casos, a vontade e o desejo do sujeito para iniciar o tratamento [2].

A internação hospitalar psiquiátrica é um momento que causa sofrimento mental para o sujeito, uma vez que impede o processo de trocas sociais, pois, ao visar à proteção do sujeito $\mathrm{e}$ seu entorno, se opera um cerceamento físico, subjetivo e dos afetos, pela deslegitimação do diálogo no momento da crise, anulando esse sujeito nas relações [3].

E o processo de reinternação é algo observado no contexto hospitalar psiquiátrico e ocasionado por diferentes motivos, entre eles ocasionados pelo sujeito (recaídas, falta de adesão ou abandono ao tratamento), pela ausência do suporte social e familiar, pelo próprio sistema de saúde (falta de medicações, falta de profissionais nos serviços da rede) [4].

Nota-se a insuficiência dos serviços substitutivos e a ineficiência da assistência, que resultam, entre outros problemas, no fenômeno da reinternação psiquiátrica [5].

Assim, o modelo de atendimento substitutivo promovido no Brasil, e que privilegia serviços extrahospitalares, se propõe a reduzir a importância do hospital no sistema como um todo [6]. Porém, a reinternação psiquiátrica, como fenômeno atual, desvela o processo ainda inconcluso e não consolidado da Reforma Psiquiátrica e confirma que a efetiva desinstitucionalização só ocorrerá com a devida substituição do modelo hospitalocêntrico pela Atenção Psicossocial [5].

$\mathrm{Na}$ atenção em saúde mental, é preciso construir estratégias e dispositivos que busquem resgatar ao sujeito, seu lugar de protagonista nas ações, fomentando a cotidianidade nos serviços de saúde e rede que reinscreva o processo de adoecimento como parte integrante da vida [3].

Estas estratégias podem ser desenvolvidas a partir da análise dos indicadores de saúde da população, entre eles os índices de hospitalização, que no contexto da saúde mental, indicam não só números, mas expressam a efetivação da Reforma Psiquiátrica.

Assim, este estudo teve como objetivo analisar os dados epidemiológicos acerca das internações hospitalares por Transtornos Mentais e Comportamentais (TMC) decorrentes do abuso do álcool e de outras substâncias psicoativas no Brasil, no decênio de 2007 a 2016, caracterizando assim as internações por dependência química no âmbito hospitalar do sistema público brasileiro.

Material e métodos

Estudo de caráter transversal, baseado em dados secundários, coletados no site do Departamento de Informática do Sistema Único de Saúde (DATASUS), no Sistema de Informações Hospitalares (SIH).

A triagem dos dados ocorreu em dezembro de 2017, a partir da plataforma Informações de Saúde (TABNET), com a seleção do item "Epidemiológicas e Morbidade" e em seguida com a escolha da opção "Morbidade Hospitalar do SUS, o que permitiu acessar o banco de dados do Sistema de Informação Hospitalar (SIH/SUS) e escolher a opção "por local de internação" no Brasil. Foram selecionadas as informações relacionadas às internações hospitalares e o capítulo V da Classificação Internacional de Doenças, 10ª Revisão (CID-10), mais precisamente, a lista de morbidades com diagnósticos de F10 (TMC devido ao uso de álcool) e de F19 (TMC devido ao uso de outras substâncias psicoativas), no decênio de 2007 a 2016. 
Foram incluídas no estudo todas as internações por TMC devido ao uso do álcool e de outras substâncias psicoativas que o SIH/SUS apresentou na busca, incluindo dados de menores de 10 anos, mesmo não sendo números significativos para considerar na discussão.

Os dados do DATASUS/TABNET/SIH foram exportados para o programa Microsoft Excel® 2016, tabulados e analisados por frequência simples. As internações hospitalares por agrupamentos da CID-10 foram analisadas com a realização de cruzamentos com ano da internação, sexo, faixa etária e Região do Brasil.

Os dados adquiridos estão disponíveis na internet, para livre consulta, disponibilizados pelo Ministério da Saúde, apresentando domínio público e preservando o sigilo dos seres humanos envolvidos, não necessitando da submissão ao Comitê de Ética em Pesquisa.

\section{Resultados}

Foram identificadas 958.635 internações hospitalares por dependência química considerando o somatório das internações por TMC devido ao uso do álcool e as internações por TMC devido ao uso de outras substâncias psicoativas.

O período de maior quantidade dessas internações foi no ano de 2011 com 108.715, representando $11,3 \%$ de todas as internações no período de 2007 a 2016.

É possível observar através do Gráfico 1 que, a partir desse período, houve um declínio no seu índice, chegando a 74.481 no último ano analisado (2016). Essa diminuição pode ser considerada positiva, pois reduziu em $30 \%$ a quantidade das internações por dependência química no Brasil.

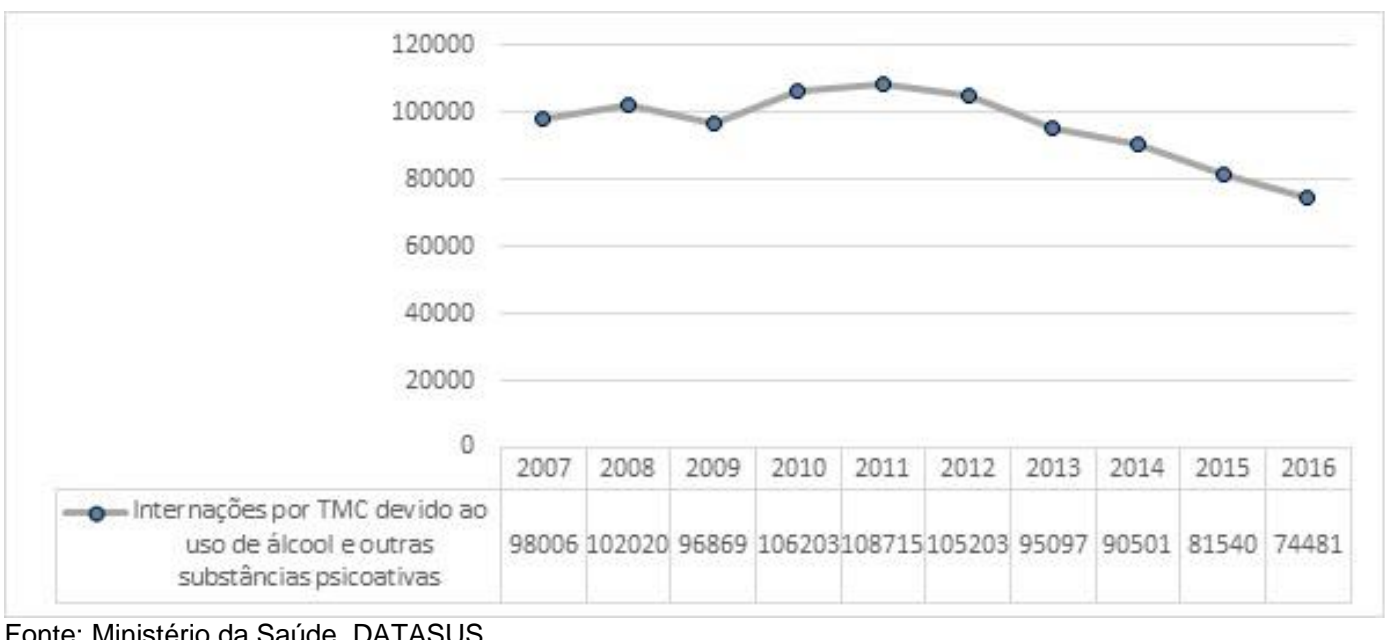

Gráfico 1 - Quantidade de internações por transtornos mentais e comportamentais devido ao uso do álcool e de outras substâncias psicoativas, por ano, no Brasil, 2007-2016.

Ao visualizar o Gráfico 2 é possível notar que houve um destaque na prevalência do sexo masculino com 818235 equivalente a $85,3 \%$ de todas as internações por dependência química no Brasil no período de 2007 a 2016.

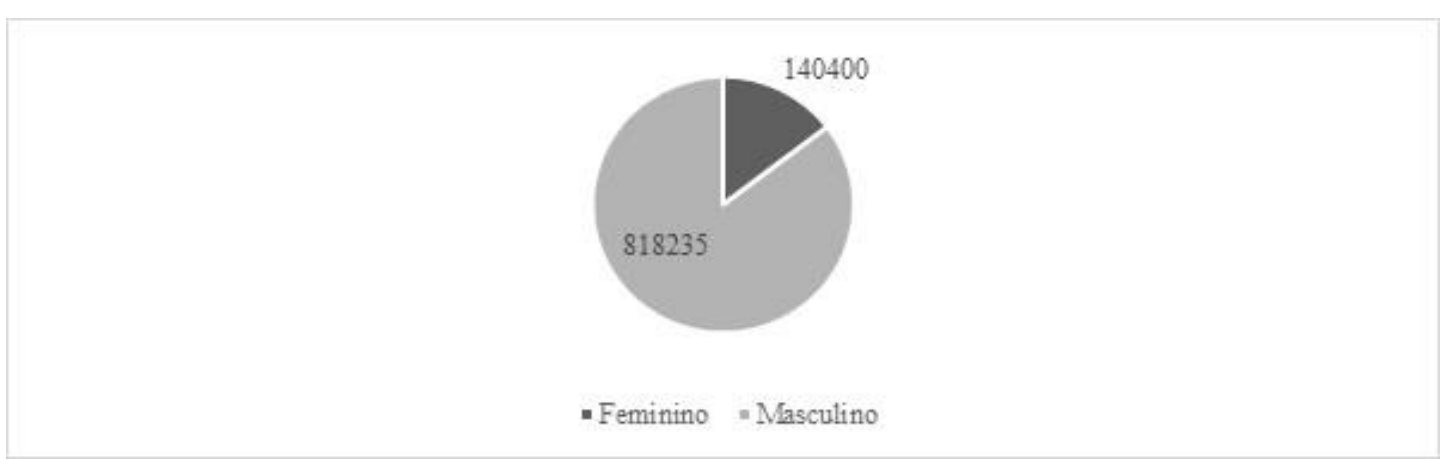

Fonte: Ministério da Saúde, DATASUS.

Gráfico 2 - Quantidade de internações por transtornos mentais e comportamentais devido ao uso do álcool e de outras substâncias psicoativas, por sexo, no Brasil, 2007-2016. 
Os dados disponíveis no DATA/SUS relacionados a esta temática exibem cortes de idades com intervalo de 4 anos, inclusive nas faixas etárias inferiores a 10 anos, porém neste estudo como forma de generalizar a faixa etária que corresponde ao grupo de crianças e adolescentes foi considerado o somatório desses dados disponíveis e categorizado em menores de 19 anos. Exibindo na totalidade quatro faixas etárias: até 19 anos, de 20 a 39 anos, de 40 a 59 anos e de 60 anos a mais. Percebeu-se que os adultos jovens na faixa etária de 20 a 39 anos apresentaram maior índice na quantidade das internações por dependência química com o total de 474.768 equivalente a quase metade do total analisado (49,5\%), conforme descrito no Gráfico 3.

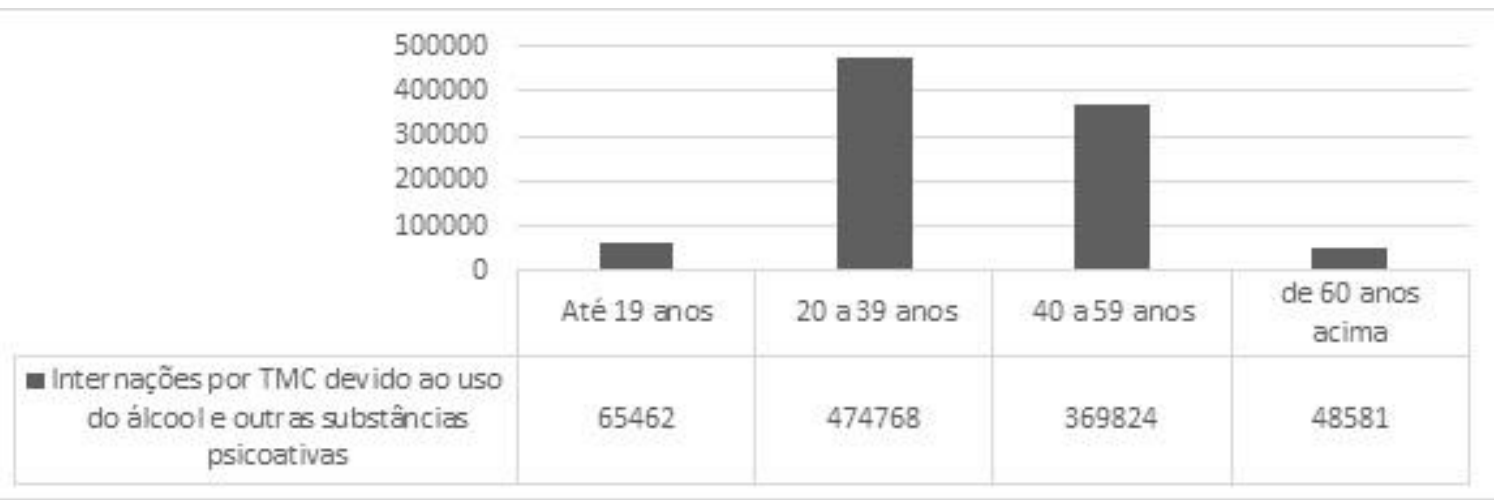

Fonte: Ministério da Saúde, DATASUS.

Gráfico 3 - Quantidade de internações por transtornos mentais e comportamentais devido ao uso do álcool e de outras substâncias psicoativas, por faixa etária, no Brasil, 2007-2016.

Através da Tabela I fica evidente que as regiões sul e sudeste apresentam os maiores índices na quantidade de internações por dependência química quando comparadas as demais regiões do país, sendo a região sudeste a que predomina com 374.472 sendo $39,1 \%$ de todas as internações avaliadas.

Tabela I - Quantidade de internações por transtornos mentais e comportamentais devido ao uso do álcool e de outras substâncias psicoativas, por região, no Brasil, 2007-2016.

\begin{tabular}{l|c|c}
\multicolumn{1}{c}{ Região } & $\mathrm{N}$ & $\%$ \\
\hline Norte & 11.644 & 1,3 \\
\hline Nordeste & 141.441 & 14,7 \\
\hline Centro - Oeste & 81.970 & 8,5 \\
\hline Sul & 349.108 & 36,4 \\
\hline Sudeste & 374.472 & 39,1
\end{tabular}

Fonte: Ministério da Saúde, DATASUS.

Discussão

A redução de $30 \%$ de internações psiquiátricas por dependência química no Brasil, no período de 2007 a 2016, pode ser considerada algo positivo na saúde pública e na questão da desinstitucionalização dos leitos psiquiátricos, pois outro estudo que abordou esta temática, na primeira década após a implantação da reforma psiquiátrica no Brasil, não identificou redução significativa nessa taxa de hospitalização, mas destacou que poderia ser reflexo do equilíbrio entre a pressão por aumento de demanda e a ampliação da capacidade de atendimento pela implantação dos CAPS e das redes locais de saúde [6].

$\mathrm{O}$ ano de 2016 também foi considerado o ano de menor índice de internações hospitalares por transtornos mentais e comportamentais em idosos brasileiros, e as internações devido ao uso do álcool e de outras substancias psicoativas representaram $25 \%$ do destas [8].

Todavia, outro estudo realizado também no Brasil, especificamente no Ceará, investigou essa mesma temática, dirigida ao público de crianças e adolescentes no período de 2000 a 2015 e não encontrou variação significativa nas taxas de hospitalização por dependência química. Este dado pode ser explicado justamente pelos resultados desta pesquisa na qual demonstrou 
que a maior quantidade deste fenômeno não está associada a faixa etária de crianças e adolescentes [9].

Em contrapartida mais um estudo, com esta mesma temática e nessa mesma primeira década (2000 a 2011) sendo apenas em um Estado brasileiro, observou aumento das taxas de internações hospitalares devido aos transtornos mentais e comportamentais por uso de álcool e outras substâncias psicoativas [10].

A predominância de internações no sexo masculino nesta pesquisa é semelhante ao estudo que avaliou os dados sociodemográficos na reinternação psiquiátrica em um Estado do Brasil e mais um estudo que avaliou esta mesma temática sendo no período de 2010 a 2012 em outro Estado brasileiro $[4,6]$.

Mais um estudo aponta a prevalência de internações masculinas, entre o período de 2012 a 2016, relacionadas à dependência química em pessoas idosas, principalmente as que são devido ao uso do álcool [8].

Foi possível identificar que a prevalência do sexo masculino em internações por dependência química está presente, mesmo no público mais jovem, conforme resultado de outra pesquisa brasileira [9].

Os adultos jovens também apresentaram prevalência na quantidade de internações em outro estudo, embora a categorização da faixa etária (30 a 49 anos) não tenha sido igual ao desta pesquisa, ainda assim representa o público jovem da fase adulta [4].

Outro estudo apresenta dados semelhantes aos encontrados nesta pesquisa, o qual evidenciou que as internações por transtornos relacionados à dependência química foram muito frequentes na população jovem com predominância da faixa etária entre 20 a 49 anos [11].

O público masculino jovem com a média de idade de 36,5 anos também foi caracterizado como predominante nas internações por intoxicação na dependência química em mais um estudo realizado no Brasil [12].

Assim como esta pesquisa evidenciou-se a região sudeste, como a região do país que apresenta o maior número de internação hospitalar por dependência química. Outro estudo que avaliou de forma geral as internações por transtorno mental comportamental em idosos também caracterizou esta mesma região como a que detém também esta maior quantidade [8].

Conclusão

Este estudo nos possibilitou compreender que as internações por dependência química no âmbito hospitalar público, no Brasil, ainda expressam números altos, mesmo tendo um declínio de mais de $30 \%$ no último analisado (2016). No entanto, esta porcentagem já tem sido um resultado favorável, na luta pela redução de hospitalizações psiquiátricas no país.

Ao relacionar os dados encontrados nesta pesquisa com outros estudos realizados no Brasil, de mesma temática, em períodos e públicos diferentes, foi possível observar que houve variação de resultados.

Os homens, mesmo em faixas etárias diferentes, são caracterizados como o público mais prevalente nestas internações hospitalares públicas para o tratamento da dependência química, o que evidencia um dado de alerta para realização de ações voltadas a saúde do homem nesta perspectiva da saúde mental.

Jovens adultos, especificamente entre 20 e 39 anos, também devem ser alvos de mais ações de saúde pública no combate ao uso do álcool e outras drogas, uma vez que eles exibiram os maiores números destas internações.

A região sudeste, igualmente, precisa fortalecer as políticas de saúde que visem à redução desse índice, garantindo rede de amparo aos usuários de drogas com o intuito de evitar a dependência química dessas pessoas.

Esses dados epidemiológicos retratam não somente informações expressas por preenchimentos de guias de informação em saúde, mas principalmente apresentam a situação de saúde, neste caso, a saúde mental da população brasileira diante do uso e abuso de drogas e ainda a necessidade de estratégias factíveis que promovam melhorias para que esse público tenha estímulo para o enfrentamento da problemática relativa ao uso de álcool e de substâncias psicoativas.

Essas estratégias podem ser discutidas e elaboradas com a participação de diferentes atores envolvidos, como os gestores públicos, os profissionais que compõem a Rede de Atenção Psicossocial (RAPS), a comunidade e os próprios usuários das substâncias psicoativas, objetivando a pactuação de métodos que alcancem resultados positivos e melhorem cada vez mais os indicadores de saúde mental. 
Como propostas de embate ao assunto em questão, torna-se possível sinalizar medidas preventivas como as ações em saúde, para que sejam inseridas com mais frequência a partir de uma responsabilização intersetorial, saúde, educação e outros, que possibilitem dialogar com os jovens e adolescentes o uso e abuso do álcool e outras drogas, tornando a base de medidas preventivas. Outra proposta seria o fortalecimento da RAPS com mais investimentos públicos financeiros, possibilitando maiores impactos nos serviços que substituem a internação hospitalar.

Sugere-se ainda uma investigação aprofundada dos números obtidos de internações por TMC em menores de 10 anos, visto que a ocorrência delas expressa uma realidade preocupante por representar crianças em situação de adoecimento psíquico resultante do acesso ao uso ou abuso de drogas.

Ressalta-se a necessidade de realização de mais estudos com esta temática no sentido de identificar o que favorece a ocorrência das internações e reinternações por TMC devido ao uso e abuso do álcool e outras substâncias psicoativas.

\section{Referências}

1. Fertig A, Schneider JF, Oliveira GC, Olschowsky A, Camatta MW, Pinho LB. Mulheres usuárias de crack: Conhecendo suas histórias de vida. Esc Anna Nery 2016;20(2):31016. https://doi.org/10.5935/1414-8145.20160042

2. Assis JT, Barreiros GB, Conceição MIG. A internação para usuários de drogas: diálogos com a reforma psiquiátrica. Rev Latinoam Psicopat Fund 2013;16(4):584-96. https://doi.org/10.1590/S1415-47142013000400007

3. Braga CP, d'Oliveira AFPL. A continuidade das internações psiquiátricas de crianças e adolescentes no cenário da Reforma Psiquiátrica Brasileira. Interface (Botucatu) 2015;19(52):33-44. https://doi.org/10.1590/1807-57622014.0227

4. Castro SA, Furegato ARF, Santos JLF. Características sociodemográficas e clínicas em reinternações psiquiátricas. Rev Latinoam Enferm 2010;18(4):800-8. Disponível em: http://www.scielo.br/scielo.php?script=sci arttext\&pid=S0104$11692010000400020 \& \operatorname{lng}=\mathrm{pt}$

5. Machado V, Santos MA. O tratamento extra-hospitalar em saúde mental na perspectiva do paciente reinternado. Psicol Estud 2013;18(4):701-12. https://doi.org/10.1590/S1413-73722013000400012.

6. Balbinot AD, Horta RL, Costa JSD, Araújo RB, Poletto S, Teixeira MB. Hospitalizações por uso de drogas não se alteram com uma década de Reforma Psiquiátrica. Rev Saúde Pública 2016;50:26. https://doi.org/10.1590/S1518-8787.2016050006085

7. Departamento de Informática do SUS - DATASUS [Internet]. Brasília, DF: Ministério da Saúde. 2008. Sistema de Informações Hospitalares - SIH. Internações hospitalares por local de internação por capítulo e lista de morbidades CID-10. [citado 2017 Mar 10]. Disponível em: http://tabnet.datasus.gov.br/cgi/deftohtm.exe?sih/cnv/niuf.def

8. Araújo RJS, Sobral JPCP, Souza RV, Soares EPS. Internações hospitalares de pessoas idosas por transtornos mentais e comportamentais no Brasil. V CIEH 2017. Disponível em: http://www.editorarealize.com.br/revistas/cieh/trabalhos/TRABALHO EV075 MD2 SA2 ID1849 01102017100622.pdf

9. Balbinoti AD. Psychiatric hospitalizations due to alcohol and drug consumption among young people in Ceará, Brazil. Clin Biomed Res 2017;37(1). Disponível em: http://seer.ufrgs.br/index.php/hcpa/article/view/67085/pdf

10. Horta RL, Costa JSD, Balbinot AD, Watte G, Teixeira VA, Poletto S. Hospitalizações psiquiátricas no Rio Grande do Sul de 2000 a 2011. Rev Bras Epidemiol 2015;18(4):918-29. https://doi.org/10.1590/1980-5497201500040019

11. Pereira PK, Santos SA, Lima LA, Legay LF, Santos JFC, Lovisi GM. Transtornos mentais e comportamentais no Sistema de Informações Hospitalares do SUS $(\mathrm{SIH}$ SUS) no estado do Rio de Janeiro no período de 1999 a 2010. Cad Saúde Coletiva 2012;20(4):482-91. https://doi.org/10.1590/S1414-462X2012000400012

12. Reis LM, Martins BF, Gavioli A, Mathias TAF, Oliveira MLF. Saúde do homem: internações hospitalares por intoxicação registradas em um centro de assistência toxicolo. Esc Anna Nery 2013;17(3):505-11. https://doi.org/10.1590/S1414$\underline{81452013000300014}$ 